\title{
The Fundamental Formula for the Area of a Triangle in Analytical Geometry.
}

\author{
By C. Twakdir.
}

(Read and Received 8th March 1912).

The algebraic methods employed are usually defective in explaining the sign to be attached to an area; while the trigonometrical method with the transformation of a trigonometrical formula has always seemed a little far fetched so early in analytical geometry.

The following algebraic demonstration is direct enough, and successfully suggests the sign to be attached.

Case I. Let $\mathrm{G}_{1}, \mathrm{G}_{2}$ be two points $\left(1, y_{1}\right)$ and $\left(1, y_{2}\right)$ on the line $x=1$.

Then the area of $O G_{1} G_{2}=\frac{1}{2} G_{1} G_{2}=\frac{1}{2}\left(y_{2}-y_{1}\right)$ in magnitude and sign.

Case II. Let $\mathrm{P}_{1}, \mathrm{P}_{2}$ be any two points $\left(x_{2}, y_{1}\right),\left(x_{2}, y_{2}\right)$, and let $O P_{1}$ and $O P_{2}$ cut the line $x=1$ in $G_{1}$ and $G_{2}$, so that $G_{1}$ and $G_{2}$ are given by $\left(1, \frac{y_{1}}{x_{1}}\right)$ and $\left(1, \frac{y_{2}}{x_{2}}\right)$, while

$$
\frac{\mathrm{OG}_{1}}{\mathrm{OP}_{1}}=\frac{1}{x_{1}}, \frac{\mathrm{OG}_{2}}{\mathrm{OP}_{2}}=\frac{1}{x_{2}} \text {. }
$$

Hence

$$
\begin{aligned}
\triangle O P_{1} P_{2} & =x_{1} x_{2} . \triangle O G_{1} G_{2} \\
& =\frac{1}{2}\left(x_{1} y_{2}-x_{2} y_{1}\right) .
\end{aligned}
$$

There remains the question of sign. When $x_{1}$ and $x_{2}$ are both like in sign, $O G_{1} G_{2}$ and $O P_{1} P_{2}$ are of like sense of rotation and $x_{1} x_{2}$ is positive. (Fig. 1). 


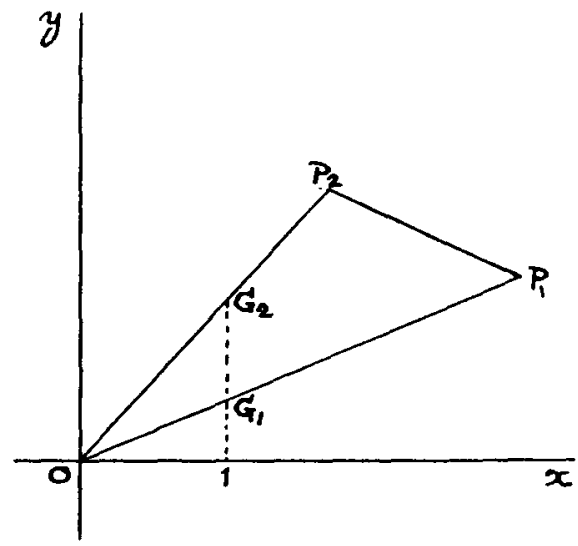

Fig. 1.

If $x_{1}$, say, is positive, and $x_{2}$ negative, then $O G_{1} G_{2}$ and $O P_{1} P_{2}$ are of opposite sense of rotation, and $x_{1} x_{2}$ is negative. (Fig. 2).

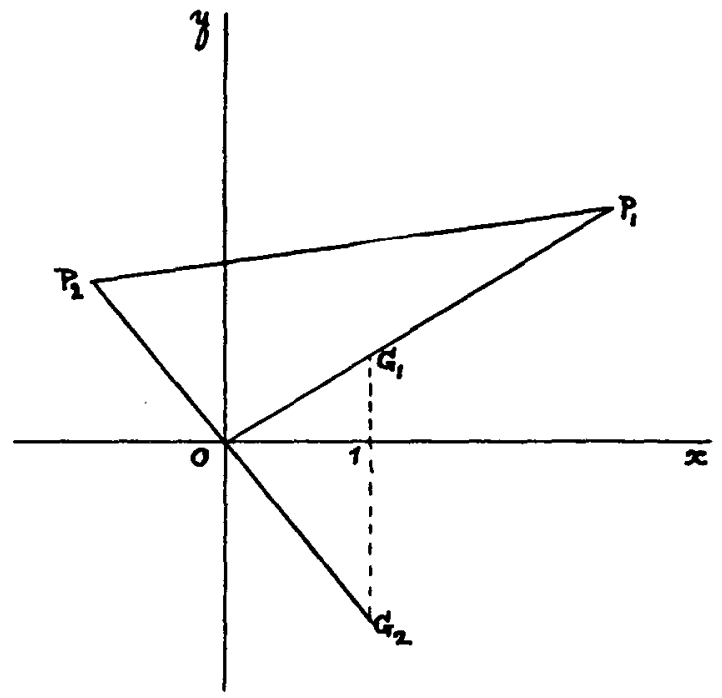

Fig. 2.

Hence in all cases the usual law for the sign of the area follows.

This is extended to the triangle $\mathbf{P}_{1} \mathbf{P}_{2} \mathbf{P}_{s}$, and to polygons, in the usual manner. 